\title{
Efeito da inundação de longa duração sob o crescimento de Pouteria glomerata (Sapotaceae), uma arbórea da várzea da Amazônia Central
}

\author{
Daniel MAURENZAㄹ Ricardo Antonio MARENCO², Maria Teresa Fernandez PIEDADE ${ }^{3}$
}

\begin{abstract}
RESUMO
Para entender a ocorrência de $P$. glomerata na várzea amazônica, investigamos as respostas morfo-fisiológicas a longo período de inundação. Durante seis meses, plântulas de $P$. glomerata foram submetidas a dois tratamentos de inundação (parcial e total) para análise da assimilação fotossintética líquida (A), eficiência quântica do fotosistema II (referido como Fv/Fm), altura, número de folhas, diâmetro do colo do caule (DCC), área foliar e biomassa da planta. Encontramos um decréscimo da atividade de trocas gasosas, das taxas de crescimento e danos foliares com o aumento do nível de inundação. Após seis meses de experimento, a área foliar, a biomassa dos órgãos vegetativos (raiz, caule e folha) e a biomassa total das plântulas inundadas foram menores que das plântulas controle, plântulas não-inundadas. De acordo com o aumento do nível de inundação, a biomassa fotoassimilada foi alocada principalmente para o caule. Somente área foliar específica, razão raiz / parte aérea e massa seca de raiz não apresentaram diferenças entre os tratamentos. As plântulas totalmente inundadas foram fortemente comprometidas, demonstrando ser esta à condição mais crítica para a manutenção do metabolismo fisiológico. $P$. glomerata foi afetada pelo longo período de inundação, no entanto a espécie revela adaptaçōes morfo-fisiologica que justifica a sua ocorrência em florestas de várzea.
\end{abstract}

PALAVRAS-ChAVE: Assimilação fotossintética, eficiência quântica do fotossistema II, biomassa, tolerância e plasticidade fenotípica.

\section{Growth of Pouteria glomerata (Sapotaceae), a tree species from the Central Amazonian floodplain, under long-term flooding}

\begin{abstract}
To understand the occurrence of $P$. glomerata in the Amazonian várzea we investigated the morpho-physiological responses to long-term flooding. Seedlings of $P$. glomerata were subjected to two flooding treatments (partial and total) for six months. Following flooding treatments, we examined light-saturated photosynthesis (A), the potential quantum yield of photosystem II (inferred as the Fv/Fm ratio), height, number of leaves (NF), stem diameter at the base of the plant (DCC), leaf area and plant biomass. We found a decrease in gas exchange parameters and growth rates, whereas leaf damage increased with flooding. After six months of flooding, leaf area, and the total plant biomass as well as the biomass of the vegetative organs (leaf, stem and root) were lower in flooded seedlings than in control, non-flooded plants. As flooding level increased assimilated carbon was mainly allocated to stems. However, flooding treatments had no significant effect on specific leaf area, the root/shoot ratio and dry mass of roots. Totally submerged roots of flooded seedlings were strongly affected, showing to be the most critical condition for maintenance of physiological metabolism. P. glomerata was affected by long-term flooding. Thus, P. glomerata exhibited adjusts morpho-fisiological which makes it possible for this species to occur in the Amazon floodplain forest.
\end{abstract}

KEY WORDS: Photosynthetic assimilation, quantum efficiency of photosystem II, biomass, and phenotypic plasticity

1 Instituto Nacional de Pesquisas da Amazônia, Max - Planck, Manaus, AM, CEP 69060-001-Brasil. E-mail: dmaurenza@yahoo.com.br

2 Instituto Nacional de Pesquisas da Amazônia, Coordenação de Pesquisas em Ciências em SilviculturaTropical, Manaus, AM, CEP 69060-001-Brasil. E-mail: rmarenco@inpa.gov.br

${ }^{3}$ Instituto Nacional de Pesquisas da Amazônia, Coordenação de Pesquisa em Biologia Aquática, Max - Planck, Manaus, AM, CEP 69060-001-Brasil. E-mail: maitepp@inpa.gov.br 


\section{INTRODUÇÃO}

No norte da América do Sul, o clima tropical com índice pluviométrico elevado e sazonal, submete os rios da bacia amazônica a um pulso de inundação regular (Junk et al., 1989). Devido à flutuação do nível da água, os rios transbordam para as margens adjacentes durante o período de cheia e leva à saturação do solo por cerca de 210 dias (Junk, 1989) por ano. Diante disso, as florestas são periodicamente alagadas e passam por alternância entre uma fase terrestre e uma fase aquática durante um ano (Junk, 1997). Nestes ambientes com inundações extremas, somente plantas que possuem eficientes adaptações para tolerar a fase aquática estão presentes. Devido à existência de poucas espécies altamente tolerantes a inundação, observa-se elevado grau de endemismo nas áreas alagáveis por rios de alta fertilidade e sedimentos em suspensão, as chamadas várzeas (Prance, 1979; Junk et al., 1989; Wittmann et al., 2006).

$\mathrm{Na}$ fase aquática, os espaços entre os sedimentos do solo são ocupados pela água, causando diversas alterações físicoquímicas edáficas (Joly e Crawford, 1982). Dentre elas, a redução de oxigênio destaca-se por limitar os processos respiratórios das raízes e por tornar o meio hipóxico ou até mesmo anóxico (Pezeshki, 2001). Para as espécies arbóreas tolerarem os efeitos do alagamento, Lobo e Joly (1998) descrevem dois tipos de mecanismos biológicos que podem ser ativados. Um deles representa as adaptaçôes para evitar a perda de oxigênio, tais como formação de lenticelas hipertrofiadas e aerênquima que permitem a entrada de oxigênio. Por outro lado, outros mecanismos como o desvio do metabolismo aeróbio para anaeróbio, redução da taxa metabólica, produção de açúcares, antioxidantes e hormônios são adaptações para tolerar o déficit de oxigênio. Maiores detalhes podem ser encontrados em Joly e Crawford (1982), Worbes et al. (1992), Crawford e Brändle (1996), Vartapetian e Jackson (1997), Pimenta et al. (1998), Waldhoff et al. (1998), De Simone et al. (2003), Rengifo et al. (2005), Oliveira-Wittmann (2007), Ferreira et al. (2007 e 2009) e Parolin (2009).

Algumas espécies apresentam mudanças morfológicas e fisiológicas como estratégia adaptativa a inundação. Sob condiçōes naturais e controladas, estudos relatam uma diminuição da assimilação fotossintética líquida em plantas alagadas (Waldhoff et al., 1998, 2002; Piedade et al., 2000; Parolin et al., 2001; Armbrüster et al., 2004; Fernandez et al., 1999). Os ajustes fisiológicos resultam em considerável impacto sobre a alocação de biomassa para órgãos vegetais como folhas, caules e raízes. Investigando espécies da várzea da Amazônia central. Parolin (2002) reporta o rápido crescimento caulinar na fase inicial da vida como maneira de aumentar a altura e escapar dos efeitos da submersão. Em termos de fenologia, algumas espécies decíduas como Albizia multiflora e Crataeva benthamii e semi-decíduas Alchornea castaneifolia e Luehea cymulosa apresentam a queda foliar total ou parcial em sincronia com o pulso de inundação (Junk et al., 1989; Schöngart et al., 2002). E ainda, o crescimento de raízes adventícias é comumente observado em plântulas inundadas (Joly e Crawford 1982, Waldhoff et al., 1998; Lopez e Kursar 2003; Parolin 2009), uma adaptação importante para absorção de oxigênio disponível na água. Em nível foliar, a análise da fluorescência da clorofila $a$ é outro meio para interpretar o desenvolvimento foliar e diagnosticar a saúde foliar. Durante a fotossíntese, a folha dissipa parte da energia solar excedente em forma de luz como estratégia de defesa. Assim, é possível identificar o nível do dano ao aparato fotossintético (Krause e Weis, 1991).

Os ajustes morfofisiológicos adaptativos dos indivíduos às mudanças ambientais, as chamadas respostas plásticas fenotípicas naturais (Schlichting, 1986), auxiliam o indivíduo ocupar seu atual ambiente de ocorrência (Shao et al., 2007). Assim, este estudo buscou avaliar o grau de plasticidade fenotípica de Pouteria glomerata, uma espécie arbórea comumente encontrada na várzea da Amazônia Central. Poucos estudos investigaram o efeito da inundação de longa duração, variando entre dois a quatro meses de experimentação (Pimenta et al., 1998; Waldhoff et al., 1998; Lopez e Kursar, 1999; Parolin, 2001; Ferreira et al., 2009). Por meio de parâmetros morfofisiológicos investigamos as adaptaçōes que a espécie expressa frente ao alagamento artificial que justifiquem a sua ocorrência nas várzeas. Assumindo que a fase aquática é um período crítico para as árvores, aceitamos como hipótese que a inundação diminui o metabolismo de $P$. glomerata. Se a hipótese estiver correta, esperamos que as respostas morfofisiológicas, em termos de trocas gasosas, fluorescencia da clorofila $a$, crescimento e biomassa, revelem as adaptaçôes de $P$. glomerata à inundação de longa duração que justifica a sua ocorrência na várzea.

\section{MATERIAL E MÉTODOS}

\section{ÁREA DE COLETA}

O material vegetal foi coletado nas margens do lago Camaleão, Ilha da Marchantaria ( $\left.3^{\circ} 15^{\prime} \mathrm{S}, 60^{\circ} 00^{\prime} \mathrm{W}\right)$, localizada próximo à confluência dos rios Solimões e Negro, $20 \mathrm{~km}$ ao sudoeste de Manaus, Amazônia Central. A altitude média do local das coletas é de $23 \mathrm{~m}$ acima do nível do mar. A precipitação anual na região é de aproximadamente $2100 \mathrm{~mm}$, com marcada sazonalidade da estação seca (junho/setembro) e chuvosa (dezembro/maio) (Parolin et al., 2001). O somatório da área de captação hídrica leva a flutuação do nível da água do local em média de $10 \mathrm{~m}$, permitindo distinguir anualmente uma fase aquática, com pico de inundação em junho/julho, e uma fase terrestre, com pico de seca em novembro/dezembro (Piedade et al., 1991, Junk et al., 1989). 


\section{OBTENÇÃO DAS PLÂNTULAS E DELINEAMENTO EXPERIMENTAL}

Plântulas de $P$. glomerata selecionadas pela similaridade em altura $(30 \pm 10 \mathrm{~cm})$ foram transplantadas para vasos $(13 \mathrm{~cm}$ de diâmetro na base, $16 \mathrm{~cm}$ na abertura e $15 \mathrm{~cm}$ de altura) contendo 2,5 L de solo do próprio local, de forma a maximizar a manutenção das condiçōes edáficas originais. Posteriormente os vasos foram transportados até as dependências do Instituto Nacional de Pesquisas da Amazônia (INPA), para três meses de aclimatação. Durante este período, todas as plântulas permaneceram sem inundação e sob sombreamento que permitiu a entrada de radiação similar à encontrada na área de coleta, cerca de $50 \%$ da radiação solar total incidente.

$\mathrm{Na}$ busca de melhor representatividade do ambiente natural, neste estudo as plântulas foram submetidas a um período de seis meses de inundação artificial, utilizando tanques $(104 \mathrm{~cm} \times 161 \mathrm{~cm})$ com capacidade para $1000 \mathrm{~L}$. Na base dos tanques foi inserido um tubo de $40 \mathrm{~mm}$ de diâmetro que permitiu controlar o nível da água. Os indivíduos foram submetidos a dois tratamentos quanto ao nível de inundação: (1) inundação parcial (IP), onde o nível de inundação foi de 2 a $3 \mathrm{~cm}$ a cima da superfície do solo; e (2) inundação total (IT), onde o nível da água permaneceu acima da gema apical da plântula e no escuro. Com o uso de placas de isopor, foi possível cobrir totalmente a abertura do tanque impedindo a entrada direta de luz. A condição de escuro foi aplicada a fim de reproduzir as condiçōes naturais durante o pico da fase aquática. Um terceiro grupo de plântulas, (3) controle (não inundado), foi irrigado diariamente.

\section{FOTOSSÍNTESE}

Medidas das taxa de assimilação fotossintética líquida (A) foram tomadas mensalmente, utilizando um sistema portátil de análise de gases por infravermelho (IRGA LI-6400, Li-Cor, Lincoln, Nebraska, EUA). O protocolo para coleta de dados foi: temperatura de $30^{\circ} \mathrm{C}$; RFA (radiação fotossinteticamente ativa) dentro da câmara de $1000 \mu \mathrm{mol} \mathrm{m}{ }^{-2} \cdot \mathrm{s}^{-1}$; umidade relativa do ar entre 70 e $80 \%$; concentração de CO atmosférico de $380 \mu \mathrm{mol} \mathrm{mol}^{-1} \mathrm{e}$ fluxo de ar de $400 \mu_{\mathrm{mol}} \mathrm{s}^{2}$. Todas as medidas foram feitas em câmara foliar com $6 \mathrm{~cm}^{2}$ de área foliar, entre 09:00 e 14:00 h. Foi utilizada uma folha por indivíduo localizada entre as 4 primeiras do ápice para a base. Nove indivíduos foram utilizados para cada tratamento.

\section{FLUORESCÊNCIA DA CLOROFILA A}

Com o uso de um fluorômetro (PEA, MK2, 9600, Hansatech, Norfolk, UK), foram tomadas mensalmente medidas de eficiência quântica do photossistema II (Fv/Fm) de nove indivíduos. Fv/Fm foi calculado a partir dos valores de fluorescência inicial $\left(F_{0}\right)$, fluorescência máxima $\left(F_{m}\right)$ e fluorescência variável $\left(\mathrm{F}_{\mathrm{v}}=\mathrm{F}_{0}-\mathrm{F}_{\mathrm{m}}\right)$. Antes de cada mediçāo, a superfície foliar foi submetida à condição de ausência de luz durante 1 hora para adaptação ao escuro.

\section{MORFOLOGIA E CRESCIMENTO}

Medidas de altura da plântula, número de folhas e diâmetro do colo caulinar (DCC) foram feitas mensalmente em nove indivíduos por tratamento. Ao final de seis meses as plântulas foram retiradas dos vasos, lavadas cuidadosamente e fracionadas em folhas, caule e raiz. Com as folhas ainda frescas, foi calculada a área foliar de cada plântula com um medidor de área foliar (modelo AM1, Delta-T Device, Cambridge, Reino Unido). O material vegetal foi seco a $95^{\circ} \mathrm{C}$ em estufa de circulação forçada (Heraeus e Memmert) até atingir peso constante e pesado em balança analítica (modelo Belmarq $210 \mathrm{~A}$ ). A partir dos dados de massa seca e área foliar, os seguintes parâmetros foram calculados para avaliar o crescimento da plântula: razão massa foliar (RMF = massa de folha $/$ massa total), razão área foliar (RAF = área foliar / massa total), área foliar específica (AFE = área foliar / massa foliar), razão massa da raiz $(\mathrm{RMR}=$ massa de raiz $/$ massa total) e razão raiz parte aérea $(\mathrm{R} / \mathrm{PA}=$ massa de raiz $/$ (massa de folha + massa de caule)).

\section{ANÁLISE ESTATÍSTICA}

Os parâmetros utilizados foram submetidos à análise de variância (ANOVA) de medidas repetidas, onde foram considerados 7 níveis dentro do fator tempo (tempo inicial + 6 meses). Quando houve diferença significativa foi aplicado o teste Tukey a posteriori para discriminar a diferença entre os tratamentos em cada mês de coleta. As análises foram conduzidas no software SYSTAT 10.2 (Wilkinson, 1998), considerando $95 \%$ de confiabilidade $(p<0,05)$.

\section{RESULTADOS}

Em todas as medidas, as plântulas sob tratamentos de inundação (parcial e total) apresentaram valores de A menores que das plântulas controle (Figura 1a). Sob IP, a assimilação fotossintética acompanhou a tendência das plântulas controles, mantendo os valores em média $1,4 \mu \mathrm{mol} \mathrm{CO}_{2}$ $\mathrm{m}^{-2} \mathrm{~s}^{-1}$ menores. A assimilação de $\mathrm{CO}_{2}$ das plântulas sob IT decresceu significativamente ao redor de $67 \%$ após 30 dias, sendo que ao final do experimento os valores foram próximos de $0 \mu \mathrm{mol} \mathrm{CO} \mathrm{m}^{-2} \mathrm{~s}^{-1}$.

A partir de 120 dias, os valores de Fv/Fm apresentaram duas respostas bem definidas: (1) plântulas fotossinteticamente danificadas, com os valores significativamente menores, ao redor de 0,70, representadas pelo tratamento IT; e (2) plântulas sem dano ao sistema fotossintético, com os valores variando entre 0,78 e 0,80 , representadas pelos tratamentos de IP e controle, respectivamente (Figura 1b)

O incremento em altura foi maior nas plântulas controles, que diferiram das demais após 90 dias (Figura 1c). Um acréscimo de $52 \%$ em 6 meses foi registrado. As plântulas sob IP e IT apresentaram alturas $24 \%$ e $38 \%$ menores que 
as plântulas controle, respectivamente. Com tendência semelhante, as plântulas controle aumentaram 122\% do número de folhas, com valores significativos aos 120 dias. Após 6 meses, as plântulas sob IP e IT apresentaram 45\% e 83\% menos folhas que as plântulas controle (Figura 1d). Diante disto, os valores da razão $\mathrm{A} / \mathrm{NF}$ das plântulas sob inundação total aumentaram significativamente aos 90 dias, sendo ao final do experimento quatro vezes maior que das plântulas controle (Figura 1e). Devido ao crescimento lento, o aumento de DCC das plântulas controles foi significativo somente aos 150 dias (Figura 1f). Quando os parâmetros foram analisados continuamente, somente DCC não foi afetado pela inundação (Tabela 1). Todos os demais parâmetros indicam tanto efeito da inundação e tempo, quanto da interação desses fatores (Tabela 1).

As análises feitas com os dados coletados ao final do experimento também demonstram mudanças no crescimento de $P$. glomerata. Nas plântulas controle a biomassa de folha correspondeu a $64 \%$ da biomassa total, enquanto a biomassa de caule $20 \%$ e a biomassa de raiz $16 \%$ (Figura 2). De acordo com o aumento do nível de inundação, a alocação de biomassa para a folha diminuiu, enquanto para o caule aumentou. Sob inundação parcial, foi observado o crescimento de uma pequena quantidade de raízes adventícias próximo à superfície
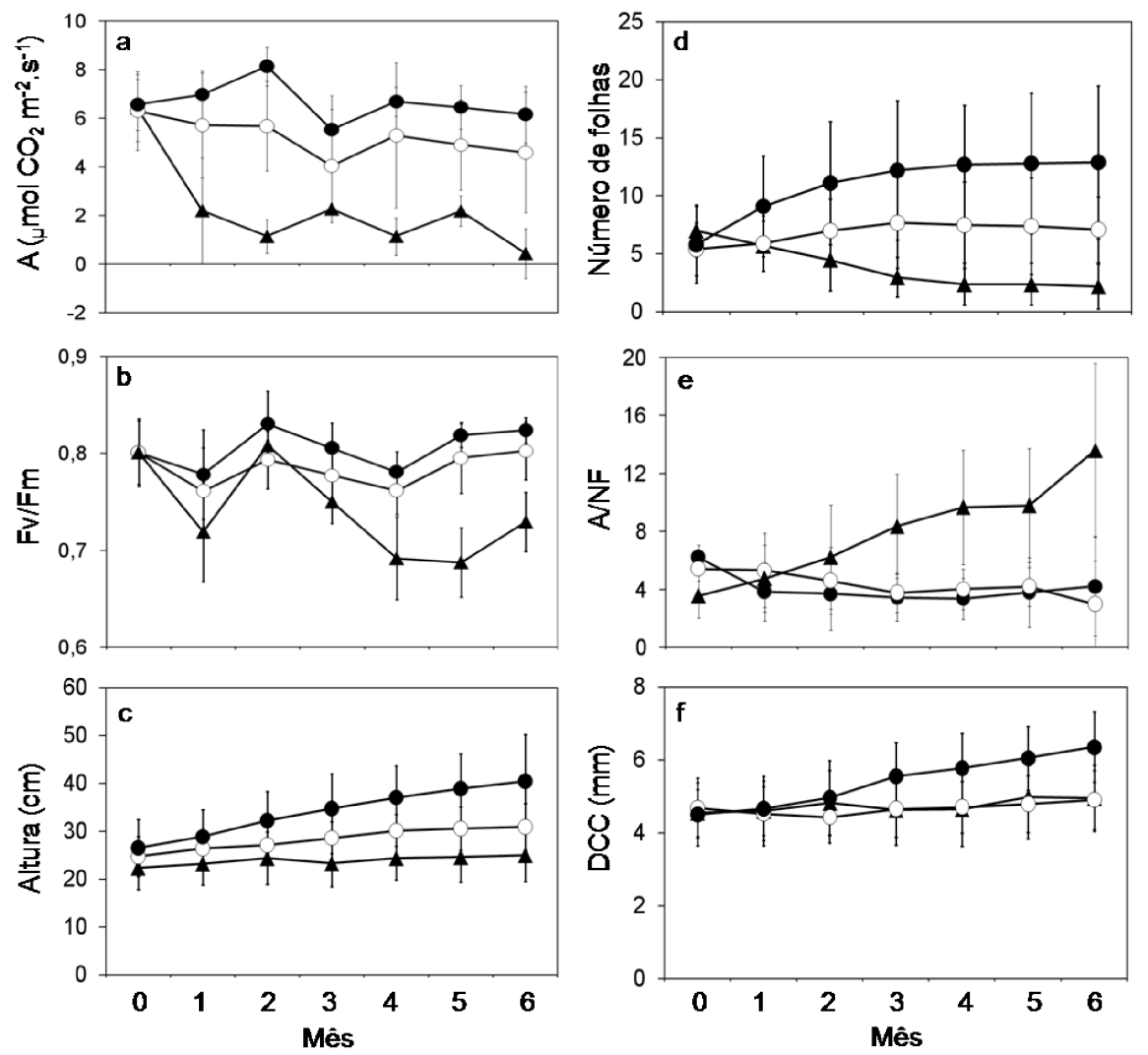

da água. Tanto os valores de biomassa como de área foliar foram significativamente menores nas plântulas inundadas (Tabela 2). Apenas os parâmetros RMF e RAF das plântulas parcialmente inundadas diferiram das plântulas controle. Por outro lado, os parâmetros $\mathrm{AFE}$, RMR e R/AP não apresentam efeito significativo da inundação.

\section{DISCUSSÃO}

As plântulas de $P$. glomerata se desenvolveram melhor nas condições não inundadas. Nos indivíduos controles foram encontrados os maiores valores de altura e número de folhas, com maior incorporação de biomassa que nas plântulas sob tratamentos de inundação. A saúde foliar foi mantida, permitindo elevado índice de eficiência quântica do FSII. Os demais parâmetros de crescimento apresentaram os maiores valores para as plântulas controle. Diante das respostas apresentadas para o período experimental avaliado, a ausência da inundação representou a melhor condição ambiental para P. glomerata. Os resultados são corroborados pelos obtidos por Worbes et al. (1992), Schluter et al. (1993) Fernandez et al., (1999) e Parolin et al., (2001) em ambiente natural, que verificaram maior produtividade na fase terrestre que durante a fase aquática, bem como os maiores valores de assimilação fotossintética das plântulas controle em outros estudos com inundação artificial (Pimenta et al., 1998; Waldhoff et al., 1998; Parolin, 2001; Lopez e Kursar, 2003; Armbrüster et al., 2004)

As plântulas que permaneceram sob inundação parcial apresentaram desenvolvimento ligeiramente menor que às plântulas controle. Sob este tratamento a espécie não produziu novas folhas, mas as manteve desde o início do experimento em bom estado fisiológico. Somente as plântulas parcialmente inundadas apresentam valores de RMF e RAF menores que as plântulas controle, isto é, pouco foi investido para o desenvolvimento foliar, mas as folhas presentes mantiveram-se saudáveis

Figura 1 - Média com desvio padrão de assimilação fotossintética líquida (a), eficiência quântica do fotosistema II (b), altura (c), número de folhas (d), razão altura número de folhas (e) e diâmetro do colo caulinar (f) durante seis meses de experimentação. Tratamentos são indicados por • controle, o inundação parcial e $\boldsymbol{\Delta}$ inundação total. 
Tabela 1 - Resultados da ANOVA de medidas repetidas (valores F) para os principais efeitos e interações dos parâmetros: Assimilação fotossintética líquida de $\mathrm{CO}_{2}(\mathrm{~A})$, eficiência quântica do fotosistema II (Fv/Fm), altura, número de folhas (NF), razão altura / número de folhas (A/NF) e diâmetro do colo caulinar (DCC). A análise usou nove indivíduos por tratamento $(n=9)$. Graus de liberdade $(\mathrm{GL})$. Os efeitos significativos são indicados com ${ }^{*}=\mathrm{P}<0,05,{ }^{* *}=$ $\mathrm{P}<0,01,{ }^{* \star *}=\mathrm{P}<0,001$.

\begin{tabular}{|c|c|c|c|c|c|c|c|}
\hline Efeito & $\mathrm{GL}$ & $A$ & $\mathrm{Fv} / \mathrm{Fm}$ & Altura & NF & $\mathrm{A} / \mathrm{NF}$ & DCC \\
\hline Inundação (I) & 2 & $43,12^{\star \star *}$ & $23,04^{\star \star *}$ & $7,56^{\star *}$ & $7,99 * *$ & $5,85^{\star \star}$ & 2,43 \\
\hline Tempo (T) & 6 & $6,81 * \star *$ & $19,65^{\star \star \star}$ & $18,56^{\star \star \star}$ & $3,32^{\star *}$ & $4,12^{\star \star \star}$ & $18,83^{\star \star *}$ \\
\hline Interacão | x T & 12 & $3,04 * * *$ & $4,09 * * *$ & 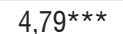 & $6,47^{\star \star *}$ & $5,01^{* * *}$ & $7,22^{* * *}$ \\
\hline
\end{tabular}

Tabela 2 - Média \pm desvio padrão e resultados da ANOVA dos parâmetros de crescimento após 6 meses de experimento. Área foliar (AF em cm), biomassa total (BT em g), biomassa da folha (BF em g), biomassa do caule (BC em g), biomassa de raiz (BR em g), razão peso foliar (RPF em g/g), razão área foliar (RAF em $\mathrm{cm} / \mathrm{g}$ ), área foliar específica (AFE em $\mathrm{cm} / \mathrm{g}$ ), razão peso raiz (RPR em g/g) e razão raiz / parte aérea (R/PA em g/g). A análise utilizou nove indivíduos por tratamento $(n=9)$. Graus de liberdade (GL); Efeito não significativo (NS). Diferença significativa, indicada pelas letras diferentes, foram detectados pelo teste Tukey considerando $95 \%$ de confiabilidade $(p<0,05)$.

\begin{tabular}{lcccccc}
\hline Parâmetros & GL & Controle & Parcial & Total & $F$ & $P$ \\
\hline AF & 2 & $5,28 \pm 2,46 \mathrm{a}$ & $1,34 \pm 0,76 \mathrm{~b}$ & $0,60 \pm 0,56 \mathrm{~b}$ & 18,73 & $<0,001$ \\
BT & 2 & $9,88 \pm 4,33 \mathrm{a}$ & $4,8 \pm 2,16 \mathrm{~b}$ & $3,42 \pm 1,10 \mathrm{~b}$ & 8,83 & $<0,01$ \\
BF & 2 & $2,79 \pm 1,34 \mathrm{a}$ & $0,81 \pm 0,63 \mathrm{~b}$ & $0,35 \pm 0,31 \mathrm{~b}$ & 13,99 & $<0,001$ \\
BC & 2 & $5,52 \pm 2,50 \mathrm{a}$ & $3,24 \pm 1,43 \mathrm{~b}$ & $2,42 \pm 0,73 \mathrm{~b}$ & 5,32 & $<0,05$ \\
BR & 2 & $1,56 \pm 0,82 \mathrm{a}$ & $0,75 \pm 0,29 \mathrm{~b}$ & $0,65 \pm 0,43 \mathrm{~b}$ & 5,95 & $<0,01$ \\
RPF & 2 & $0,280 \pm 0,083 \mathrm{a}$ & $0,165 \pm 0,077 \mathrm{~b}$ & $0,142 \pm 0,029 \mathrm{ab}$ & 7,04 & $<0,01$ \\
RAF & 2 & $0,574 \pm 0,340 \mathrm{a}$ & $0,239 \pm 0,102 \mathrm{~b}$ & $0,246 \pm 0,058 \mathrm{ab}$ & 5,08 & $<0,05$ \\
AFE & 2 & $1,99 \pm 0,670 \mathrm{a}$ & $1,54 \pm 0,28 \mathrm{a}$ & $1,72 \pm 0,06 \mathrm{a}$ & 1,98 & NS \\
RPR & 2 & $0,160 \pm 0,046 \mathrm{a}$ & $0,170 \pm 0,050 \mathrm{a}$ & $0,180 \pm 0,075 \mathrm{a}$ & 0,21 & $\mathrm{NS}$ \\
R/PA & 2 & $0,194 \pm 0,066 \mathrm{a}$ & $0,209 \pm 0,073 \mathrm{a}$ & $0,228 \pm 0,121 \mathrm{a}$ & 0,27 & $\mathrm{NS}$ \\
\hline
\end{tabular}

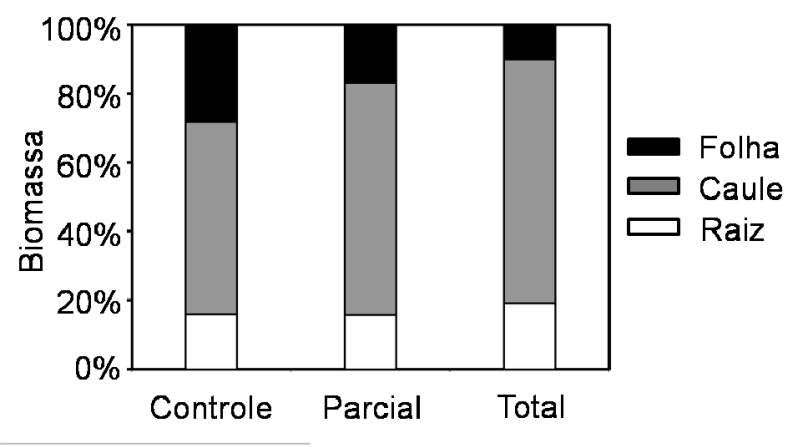

Figura 2 - Porcentagem de biomassa (em g) alocada para as frações da planta nos tratamentos de inundação após seis meses.

e garantiram a entrada de energia para a planta. A maioria dos parâmetros utilizados indica valores intermediários das plântulas sob IP em relação aos demais tratamentos, porem, a assimilação fotossintética líquida e a eficiência quântica do FSII foram elevados. Assim, a inundação parcial conduziu a pequeno decréscimo do metabolismo de P. glomerata, com a manutenção da integridade do aparato fotossintético.

Os mecanismos evidenciados apresentaram-se como ajustes ao alagamento. Isto ocorre porque quando a água satura o solo, as folhas fecham os estômatos pela falta de oxigênio. Embora haja desacordos, este comportamento ocorre porque o alagamento pode estimular a produção de ácido abscísico
(Else et al., 1996; Vartapetian e Jackson, 1997), um hormônio que reconhecidamente estimula o fechamento dos estômatos. Sob alagamento, os espaços entre os sedimentos que compõe o solo são ocupados o que bloqueia as trocas gasosas na interface raiz - atmosfera. Assim, o potencial redox do solo diminui e a capacidade e realizar trocas iônicas com as raízes torna-se insuficiente (Pezeshki, 2001), de modo que a oxigenação das raízes e de outros órgãos das plantas essencialmente aeróbios é interrompida (Crawford e Beaendle, 1996; Visser et al., 2003). Para auxiliar na manutenção do metabolismo, outros mecanismos podem ter atuando conjuntamente, como a observada produção de raízes adventícias. Este comportamento também foi reportado para diversas espécies de áreas alagáveis, (Lobo e Joly, 1998; Pimenta et al., 1998; Waldhoff et al., 1998; Chen et al. 2002; Armbrüster et al., 2004). Por exemplo, raízes adventícias de Senna reticulata alcançaram $150 \mathrm{~cm}$ em 4 meses sob inundação parcial (Parolin 2001). Embora a produção de massa de raízes adventícias foi pequena, possivelmente tiveram grande importância do ponto de vista funcional, pois essas raizes promovem a entrada de oxigênio e nutrientes necessários para as folhas e para a raiz primária durante a fase aquática (De Simone et al., 2003).

Quando as plântulas foram submetidas à submersão, a espécie assimilou menos $\mathrm{CO}_{2}$, reduziu o número de folhas, o incremento em altura e DCC. Uma diminuição acentuada 
da produtividade foi registrada aos 30 dias. Após seis meses, a área foliar e biomassa dos órgãos folha, caule e raiz das plantulas sob submersão foram menores que as plantulas controle e sob inundação parcial. Notadamente, $P$. glomerata diminuiu a produtividade como resposta a submersão, o que comprometeu o crescimento dos órgãos vegetativos. Em nível foliar, um decréscimo de Fv/Fm revelou danos foliares apenas a partir de 120 dias. Em estudo com Symmeria paniculata, uma espécies típica das áreas alagáveis amazônicas, Waldholff et al., (2002) descartam o efeito do nível de inundação, mas corrobora com nossos resultado onde encontrou uma diminuição de Fv/Fm em folhas submersas por longo periodo (a cima de $1 \mathrm{~m}$ de profundidade, correspondente ao máximo de 163 dias de submersão). Para a sobrevivência da espécie, outros ajustes em nível molecular podem atuar juntamente com as alteraçōes morfofisiológicas. Para a região amazônica, De Simone et al. (2003) reportam a produção de reservas de carboidratos durante a fase terrestre para as plantas garantirem estoque de energia suficiente para a fase aquática. Em estudos com Himathantus sucuuba (Ferreira et al., 2009) são relatadas dormência e o desvio do metabolismo aeróbio para anaeróbio em plantas submersas, com produção de álcool desidrogenase, uma enzima chave para a fermentação alcoólica, o que resulta em produção de energia e liberação de etanol. Outros mecanismos como produção de antioxidantes (OliveiraWittmann, 2007) pode estar envolvido e são sugeridos para futuras investigaçôes.

Para $P$. glomerata, a inundação total mostra-se claramente como a situação mais crítica. Em condições naturais, um somatório de diversas alterações de natureza física atua durante a submersão, tais como: (1) a imediata interrupção na interface planta-atmosfera, e conseqüentemente, das condiçōes para troca gasosa das folhas. Ou seja, a disponibilidade de oxigênio é extremamente baixa tanto para as raízes como para as outras partes da planta, conforme foi detectado no primeiro mês; (2) o decréscimo da disponibilidade de luz de acordo com o aumento da profundidade. Para os rios da Amazônia, em particular os de água branca, este efeito é acentuado. Usando como metodologia o disco de Secchi, Furch (1997) mediu a entrada de luz nas águas que inundam a mesma área de coleta do presente estudo até $30-50 \mathrm{~cm}$ de profundidade. Devido à alta quantidade de sedimentos em suspensão, que é característica dos rios de água branca, a luz, como um recurso essencial para a entrada de energia ao sistema fotossintético, é ausente para uma plântula que esta sob sete metros de coluna de água; (3) a coluna da água é profunda, principalmente nas cotas mais baixa de inundação. $\mathrm{O}$ peso da água exerce alta pressão sobre o tecido vegetal, causando alteraçôes nas diferenças de pressão necessária para funcionamento fisiológico; e (4) a alta quantidade de sedimentos em suspensão na água e que se depositam na superfície foliar, criando outra barreira para as trocas gasosas.
Do total de 16 parâmetros utilizados neste estudo, 12 mostraram o efeito significativo negativo da inundação. Nenhuma morte foi registrada durante seis meses de experimentação, até mesmo das plântulas sob tratamento de IT. Assim aceitamos a hipótese de diminuição do metabolismo aeróbio de $P$. glomerata durante a fase aquática. Entretanto, neste estudo também tornaram-se evidentes as adaptaçôes morfofisiológicas da espécie à inundação parcial e total, tais como redução das trocas gasosas do sistema fotossintético e alocação distinta dos produtos fotoassimilados. Em virtude dos resultados apresentados, $P$. glomerata demonstra alta plasticidade fenotípica que permite tolerar até mesmo seis meses de inundação total, o que justifica a sua ocorrência nas várzeas.

\section{BIBLIOGRAFIA CITADA}

Armbrüster, N.; Müller, E.; Parolin, P. 2004. Constricting responses of two Amazonian floodplain trees to hidrological changes. Ecotropica, 10(2): 73-84.

Chen, H.; Qualls, R.G.; Miller, G.C. 2002. Adaptive responses of Lepidium latifolium to soil flooding: biomass allocation, adventitious rooting, aerenchyma formation and ethylene production. Environmental and Experimental Botany, 48: 119-128.

Crawford, R.M.M; Braendle, R. 1996. Oxygen deprivation stress in a changing environment. Journal of Experimental Botany, 47(295): 145-159.

De Simone, O.; Junk, W.J; Schmidt, W. 2003. Central Amazon Floodplain Forest: Root Adaptations to Prolonged Flooding. Russian Journal of Plant Physiology, 50(6): 943-951.

Else, M.A.; Tiekstra, A.E.; Croker, S.J.; Davies, W.J.; Jackson, M.B. 1996. Stomatal closure in flooded tomato plants involves abscisic acid and a chemically unidentified anti-transpirant in xylem sap. Plant Physiology, 112(1): 239-247.

Fernandez, M.D.; Pieters, A.; Donoso, C.; Herrera, C.; Tezara, W. 1999. Seasonal changes in photosynthesis of trees in the flooded forest of the Mapire River. Tree Physiology, 19: 79-85.

Ferreira, C.S.; Piedade, M.T.F.; Franco, A.C.; Gonçalves, J.F.C.; Junk, W.J. 2009. Adaptive strategies to tolerate prolonged flooding in seedlings of floodplain and upland populations of Himatanthus sucuuba, a Central Amazon tree. Aquatic Botany, 90: 246-252.

Ferreira, C.S.; Piedade, M.T.F.; Junk, W.J.; Parolin, P.; 2007. Floodplain and upland populations of Amazonian Himatanthus sucuuba: effects of flooding on germination, seedling growth and mortality. Environmental and Experimental Botany, 60: 477-483.

Furch, K. 1997. Chemistry of várzea and igapó soils nutrient inventory of their floodplain forest. In: Junk, W.J. (Ed.). The Central Amazonian Floodplain: Ecology of a Pulsing Systems. Springer, Berlin. p. 47-67.

Joly, C.A.; Crawford, R.M.M. 1982. Variation in tolerance and metabolic responses to flooding in some tropical trees. Journal of Experimental Botany, 33(135): 799-809. 
Junk, W.J. 1989. Flood tolerance and tree distribution in central Amazonian floodplains. In: Holm-Nielsen, LbB.; Nielsen, I.C. e Balslev, H. (Eds). Tropical Forest: botanical dynamics, speciation and diversity. Academic Press, New York. P. 47-64.

Junk, W.J. 1997. Structure and Function of the Large Central Amazonian River Floodplains: Synthesis and Discussion. In: Junk, W.J. (Ed.). The Central Amazonian Floodplain: Ecology of a Pulsing Systems. Springer, Berlin. p. 3-20.

Junk, W.J.; Bayley, P.B.; Sparks, R.E. 1989. The flood pulse concept in river-floodplain systems. Canadian Special Publication of Fisheries end Aquatic Science, 106: 110-127.

Krause, G.H.; Weis, E. 1991. Chlorophyll fluorescence and photosynthesis: the basics. Annual Review of Plant Physiology and Plant Molecular Biology. 42: 313-349.

Lobo, P.C.; Joly, C.A. 1998. Tolerance to hypoxia and anoxia in neotropical tree species. In: Scarano, F.R.; Franco, A.C. (Eds). Ecophysiological strategies of xerophytic and amphibious plants in the neotropics. PPGE-UFRJ, Rio de Janeiro, Brazil. p. 137-156.

Lopez, O.R.; Kursar, T.A. 1999. Flood tolerance of four tropical tree species. Tree physiology, 19: 925-932.

Lopez, O.R.; Kursar, T.A. 2003. Does flood tolerance explain tree species distribution in tropical seasonally flooded habitats?. Oecologia, 136: 193-204.

Oliveira-Wittmann, A. 2007. Conteúdo de tococromanóis em espécies arbóreas de várzea da Amazônia Central sob condiçôes controladas. Tese de doutorado, Instituto Nacional de Pesquisas da Amazônia/Universidade Federal do Amazonas, Manaus, Amazonas. 148pp.

Parolin, P. 2001. Morphological and physiological adjustments to waterlogging and drought in seedlings of Amazonian flooplain trees. Oecologia, 128: 326-335.

Parolin, P. 2009. Submerged in darkness: adaptations to prolonged submergence by woody species of the Amazonian floodplain. Annals of Botany, 103: 359-376.

Parolin, P.; Junk, W.J.; Piedade, M.T.F. 2001. Gas exchange of six tree species from Central Amazonian floodplains. Tropical Ecology, 42: 15-24.

Pezeshki, S.R. 2001. Wetland plant responses to soil flooding. Environmental and Experimental Botany, 46: 299-312.

Piedade, M.T.F.; Junk, W.J.; Long, S.P. 1991. The productivity of the $\mathrm{C}$ grass Echinochloa polystachya on the Amazon floodplain. Ecology, 72(4): 1456-1463.

Piedade, M.T.F.; Junk, W.J.; Parolin, P. 2000. The flood pulse and photosyhthetic response of trees in a white water floodplain (Várzea) of the Central Amazon, Brazil. Verh. Internat. Verein. Limnol, 27: 1734-1739.

Pimenta, J.A.; Bianchini, E.; Medri. M.E. 1998. Adaptations to flooding by tropical trees: morphological and anatomical modifications. In: Scarano, F.R.; Franco, A.C. (Eds). Ecophysiological strategies of xerophytic and amphibious plants in the neotropics. PPGE-UFRJ, Rio de Janeiro, Brazil. p. 157-176.
Prance, G.T. 1979. Notes on the vegetation of Amazonia III. The terminology of Amazonian forest types subject to inundation. Brittonia, 31: 26-38.

Rengifo, E.; Tezara, W.; Herrera, A. 2005. Water relations, Chlorophyll a fluorescence, and contents of saccharides in tree species of a tropical forest in response to flood. Photosynthetica, 43(2): 203-210.

Schlichting, C.D. 1986. The evolution of phonotypic plasticity in plants. Annual Review of Ecology and Systematics, 17: 667-693.

Schlüter, U.B.; Furch, B.; Joly, C.A. 1993. Physiological and anatomical adaptations of young plants of Astrocarium jauari to periodic and long term inundation of the floodplain biotopes of Central Amazonia.Biotropica, 25(4): 384-396

Schöngart, J.; Piedade, M.T.F.; Ludwigshausen, S.; Horna, V.; Worbes, M. 2002. Phenology and stem-growth periodicity of tree species in Amazonian floodplain forests. Journal of Tropical Ecology, 18: 581-597.

Shao, H.B.; Guo, Q.J.; Chu, L.Y.; Zhao, X.N.; Su, Z.L.; Hu, Y.C.; Cheng, J.F. 2007. Undertanding molecular mechanism of higher plant plasticity under abiotic stress. Colloids and Surfaces B: Biointerfaces, 54: 37-45.

Vartapetian, B.B.; Jackson, M.B. 1997. Plant adaptations to anaerobic stress. Annals of Botany, 79 (supplement A): 3-20.

Visser, E.J.W.; Voesenek, L.A.C.J.; Vartapetian, B.B.; Jackson, M.B. 2003. Flooding and plant growth. Annals of Botany, 91:107-109

Waldhoff, D.; Furch, B.; Junk, W.J. 2002. Fluorescence parameters, chlorophyll concentration, and anatomical features as indicators for flood adaptation of an abundant tree species in Central Amazonia: Symmeria paniculata. Envionmental and Experimental Botany, 48: 225-235.

Waldhoff, D.; Junk, W.J.; Furch, B. 1998. Responses of three central Amazonian tree species to drought and flooding under controlled conditions. International Journal of Ecology and Environment Science, 24:2 37-252.

Wilkinson, L. 1998. Systat: the system for statistics. Systat Inc. Evanston, Illinois, USA.

Wittmann, F.; Schöngart, J.; Montero, J.C.; Motzer, T.; Junk, W.J.; Piedade, M.T.F.; Queiroz H.L.; Worbes, M. 2006. Tree species composition and diversity gradients in white-water forest across the Amazon basis. Journal of biogeography, 33: 1334-1347.

Worbes, M., Klinge, H., Revilla, J.D.; Martius, C. 1992. On the dynamics, floristic subdivision and geographical distribution of várzea forest in central Amazonia. Journal of vegetable science, 3: $553-564$

Recebido em 28/11/2008

Aceite em 06/07/2009 
